\title{
Accelerated Weathering of Polyvinyl Chloride-based Wood-plastic Composites: Effect of Plant Species
}

\author{
Ruige Qi, ${ }^{\mathrm{a}}$ Chunxia He, ${ }^{\mathrm{a}, *}$ and Qiang Jin ${ }^{\mathrm{b}}$ \\ The effect of plant species on the accelerated weathering behaviors of \\ polyvinyl chloride-based wood-plastic composites (WPCs) was studied. \\ The selected plant species were eucalyptus, rice husk, and bamboo. The \\ color and chemical compositional changes that occurred due to \\ accelerated weathering were monitored using colorimetry and Fourier \\ transform infrared spectroscopy. The lignin and carbonyl contents of the \\ WPCs were altered with exposure. The color change and lightness of the \\ weathered WPCs increased with exposure time, and the degree of \\ increase depended on the plant species. The water absorption and \\ swelling ratio of the WPCs increased with an increase in exposure. The \\ eucalyptus-based composite was the highest ranked in terms of \\ mechanical properties, and the microstructure of the impact section \\ showed that the interfacial bonding performance deteriorated after \\ exposure. In conclusion, the selection of plant fibers is critical to the \\ service performance of WPCs.
}

Keywords: Polyvinyl chloride; Plant species; Accelerated weathering; Infrared spectroscopy

Contact information: a: College of Engineering, Nanjing Agricultural University, Key Laboratory of Intelligent Agricultural Equipment in Jiangsu Province, Nanjing 210031, China; b: College of Water Resources and Civil Engineering, Xinjiang Agricultural University, Urumchi, 830001, China;

* Corresponding author: chunxiahe@tom.com

\section{INTRODUCTION}

Wood-plastic composites (WPCs) are a product class comprising a wood component and a polymer matrix. Their excellent properties, in comparison with those of solid wood, have led to a broad range of applications in many fields (Dou et al. 2015; Guan and $\mathrm{Li}$ 2018). However, widespread use has been limited due to an insufficient understanding of the weathering of WPCs (Chan et al. 2020). Although incorporation of plant fibers into plastics improves the mechanical properties of the resulting composite material relative to pure plastics, this has an adverse effect on the service life of the material due to swelling and degradation (Gao et al. 2016).

Traditionally, WPC production has focused on the use of a few selected wood species, and relatively little information is available on the effect of various plant species (such as husk) on the properties. The influence of wood species on the mechanical, interface, and dimensional stability properties of WPCs, as well as on their microstructure, have previously been reported (Peng et al. 2014; Nguyen et al. 2020). In addition, the effect of the wood species on the mechanical, thermal, and accelerated weathering behaviors of high-density polyethylene-based WPCs has been investigated (Fabiyi and McDonald 2010). A limitation of the previous work is that it did not compare the effect of the different plant species on water absorption, mechanical properties, and the interface bonding ability of WPCs before and after weathering (Temiz et al. 2007). A WPC that is exposed to the environment will be degraded by the presence of ultraviolet (UV) radiation, heat, oxygen, 
and water (Krzysztof et al. 2019). These environmental conditions in turn affect the internal and surface structures, as well as the color of the WPC. Results can include a loss of the aesthetic character of the material, and potential in-service failure.

Therefore, the objective of this study was to evaluate the effects of plant fibers from different plant species on the aging performance of WPCs by simulating long-term aging. The WPCs are characterized by their mechanical properties, surface color changes, characteristic functional group changes, tensile fracture surface analysis, water absorption, and swelling ratio. The results and discussions provide a theoretical basis for future applications in the design of anti-aging WPCs.

\section{EXPERIMENTAL}

\section{Materials}

Polyvinyl chloride (PVC) SG-5 was purchased from Xinjiang Tianye Group Co., Ltd., Urumchi, China. Eucalyptus, rice husk, and bamboo materials were also obtained from Xinjiang, Urumchi, China. Table 2 shows the comparison of the contents of cellulose, hemicellulose, lignin, and ash of these fibers reported (Petchwattana et al. 2017). Other materials used were H-108 PE wax and non-toxic $603 \mathrm{Ca} / \mathrm{Zn}$ composite stabilizers, both purchased from Shanghai Wenhua Chemical Pigment Co., Ltd., Shanghai, China. Maleic anhydride grafted polyvinyl chloride (MAH-PVC) was purchased from Dongguan Lok Wah Plastic Chemical Co., Ltd. (Dongguan, China).

Table 1. Contents of the Three Main Chemical Components of Plant Fibers

\begin{tabular}{|c|c|c|l|l|}
\hline ID & Cellulose (\%) & Hemicellulose (\%) & Lignin (\%) & Ash (\%) \\
\hline Eucalyptus & 42.72 & 17.69 & 23.99 & 4.22 \\
\hline Bamboo & 48.78 & 18.21 & 15.6 & 3.53 \\
\hline Rice husk & 43.59 & 15.30 & 20.48 & 6.61 \\
\hline
\end{tabular}

\section{Composites Preparation}

Eucalyptus, rice husk, and bamboo materials were initially crushed and subsequently ground to pass through a 100-mesh screen, and then dried at $90{ }^{\circ} \mathrm{C}$ for $12 \mathrm{~h}$ in a DHG-9140A electro-thermostatic drum-wind drying oven (Nanjing Dongmai Scientific Instrument Co., Ltd., Nanjing, China).

As shown in Table 1, the powders of eucalyptus, rice husk, and bamboo were uniformly mixed with MAH-PVC and $\mathrm{Ca} / \mathrm{Zn}$ stabilizer and then dried at $105^{\circ} \mathrm{C}$ for $4 \mathrm{~h}$, again mixed with a certain proportion of PVC, and then extruded (RM200C Taber Twin Screw Extruder; Harbin Hapu Electrical Technology Co., Ltd., Harbin, China). The extruded fibers were cut it into $100 \mathrm{~mm} \times 10 \mathrm{~mm} \times 7 \mathrm{~mm}$ samples and then placed in a xenon lamp accelerated aging test chamber.

Table 2. Sample Composition

\begin{tabular}{|c|c|c|c|c|c|c|c|}
\hline \multirow{2}{*}{ Sample ID } & \multicolumn{7}{|c|}{ Mass Ratio } \\
\cline { 2 - 8 } & EF & RHF & BF & PVC & MAH-PVC & Stabilizer & Wax \\
\hline EWPC & 50 & 0 & 0 & 50 & 1.5 & 4 & 2.5 \\
RWPC & 0 & 50 & 0 & 50 & 1.5 & 4 & 2.5 \\
BWPC & 0 & 0 & 50 & 50 & 1.5 & 4 & 2.5 \\
\hline
\end{tabular}




\section{Characterization}

Accelerated xenon lamp artificial aging

In order to evaluate the durability of the studied WPCs, an artificial weathering test was conducted following the standard method reported in GB/T 16422.3 (2014). An Atlas Ci3000+ xenon lamp (Shenzhen Kewen Environmental Test Instrument Co., Ltd., Shenzhen, China) was used. The severe conditions for climatic aging were simulated. A $4500 \mathrm{~W}$ water-cooled xenon lamp was used as light source, which provided radiation at wavelengths of 290 to $800 \mathrm{~nm}$. One cycle was conducted every $2 \mathrm{~h}$, with $102 \mathrm{~min}$ of illumination time and $18 \mathrm{~min}$ of rain time without illumination. The relative humidity of the light period was set at $60 \pm 5 \%$, and the rain period was at $50 \pm 5 \%$. The radiant intensity and total aging time of the sample were $550 \mathrm{~W}$ and $960 \mathrm{~h}$, respectively. After exposure, the samples were removed to investigate the mechanical properties, surface morphology, and chemical composition.

\section{Chemical compositional analysis of composites}

A Nicolet iS10 Fourier-transform infrared (FTIR) spectrometer (ThermoFisher Scientific Co., Ltd., Beijing, China) was used to observe the chemical structural changes in the composites. The equipment was operated in the range of $400 \mathrm{~cm}^{-1}$ to $4000 \mathrm{~cm}^{-1}$ with a resolution of $4 \mathrm{~cm}^{-1}$, and each spectrum was gathered using 16 scans in absorbance mode.

The WPC degradation was studied by following carbonyl index and lignin index, which were calculated using Eqs. 1 and 2,

Carbonyl index $=I_{\mathrm{c}} / I_{\mathrm{r}}$

Lignin index $=I_{1} / I_{\mathrm{R}}$

where $I_{\mathrm{c}}$ and $I_{1}$ are the intensities of the carbonyl and lignin absorption bands (1700 to 1800 $\mathrm{cm}^{-1} ; 1593$ to $1597 \mathrm{~cm}^{-1}$, respectively), and $I_{\mathrm{R}}$ is the intensity of the reference band (2900 to $2950 \mathrm{~cm}^{-1}$ ). The reference band was not affected by UV irradiation.

\section{Water absorption and swelling radio}

The water absorption (WA) test was performed in accordance with the GB/T 17657 (2013) standard. Before the test, the samples were oven-dried at $90{ }^{\circ} \mathrm{C}$ for $12 \mathrm{~h}$. Subsequently, the samples for the WA test were immersed in deionized water at $23 \pm 1{ }^{\circ} \mathrm{C}$ for $24 \mathrm{~h}$ in a thermostatic water tank. The WA values were calculated based on the weight percent gains after removing excess water on the exterior surface.

The prepared sample was marked along the center line of length and width, and the dimensional change of the spline thickness direction before and after immersion was measured with a digital display verdier caliper (accurate to $0.01 \mathrm{~mm}$ ) on the streamed part. The swelling ratio of the sample was calculated according to Eq. 3,

$$
\mathrm{SR}=\frac{L_{2}-L_{1}}{L_{1}} \times 100 \%
$$

where $L_{1}$ is the size of the spline before immersion, and $L_{2}$ is the size of the spline after immersion. 


\section{Color change}

The color of the samples was characterized using an HP-200 precise color meter (Shanghai Chinaspec Optoelectronics Technology Co., Ltd., Shanghai, China), which reported color coordinates in the CIE 1976 color space $\left(L^{*} a * b^{*}\right)$,

$$
\Delta E=\left(\Delta L^{* 2}+\Delta a^{* 2}+\Delta b^{* 2}\right)^{\frac{1}{2}}
$$

where $\Delta E, \Delta L, \Delta a$, and $\Delta b$ represented the difference in color $(E)$, color lightness $\left(L^{*}\right)$, red to green $\left(a^{*}\right)$, and yellow to blue $\left(b^{*}\right)$. Five characterization measurements per sample were made and the average value was reported. Relative lightness $(\Delta L)$ was used to understand the degree of lightness of weathered WPC relative to the original WPC color. The total color change $(\Delta E)$ characterizes the anti-aging properties of the WPC.

\section{Mechanical properties}

The mechanical properties of composites were measured using a CMT6104 electronic universal testing machine (MTS Industrial Systems Co., Ltd., Shenzhen, China). The tensile and flexural strengths of composites were measured according to GB/T 1040.1 (2006) and GB/T9341 (2008) standards, respectively. The sample size was $1000 \mathrm{~mm} \times 10$ $\mathrm{mm} \times 70 \mathrm{~mm}$. The test loading speed used was $2.0 \mathrm{~mm} / \mathrm{min}$, and all measurements were carried out at room temperature. Meanwhile, each group of tests was performed five times and the average value was taken to reduce the error.

\section{Microscopic structure of composites}

Analysis of the internal structure of the composites was performed using a S-4800 scanning electron microcope (SEM, Hitachi, Tokyo, Japan). Prior to the SEM analysis, the surfaces of the composites were sprayed with gold using an E-1010 ion sputter coater (Hitachi, Tokyo, Japan).

\section{Statistical Analysis}

Mean differences among the variables (plant species or plant species and exposure time) of the WPC on the chemical composition, color change, and mechanical properties of the un-weathered and weathered WPC were conducted using analysis of variance (SPSS_20.0, IBM Corp., Armonk, NY, USA). Additionally, a follow-up test was performed for the significant parameters using a Scheffe test, and the p-values were reported.

\section{RESULTS AND DISCUSSION}

\section{Surface Chemistry}

The nature of the chemical groups can be directly analyzed by FTIR. Figure 1 (a) and (b) shows the chemical changes that occurred before and after aging the composites obtained from different plant species. The band ranging from 3300 to $3500 \mathrm{~cm}^{-1}$ was assigned to the stretching vibration of hydroxyl group in the molecule (Paul et al. 2009); its value was found to increase with aging. This result showed that the hydroxyl content was increased, i.e., water absorption of the three WPCs was increased. The band ranging from 1700 to $1735 \mathrm{~cm}^{-1}$ was assigned to the stretching vibration of $-\mathrm{C}=\mathrm{O}$ group in carboxylic ester compounds (related to lignin) and ketone compounds (Cavdar et al. 2018). 
This bands decreased due to aging, which revealed that the lignin broke down. The peak at $1650 \mathrm{~cm}^{-1}$ was assigned to $-\mathrm{C}=\mathrm{C}$ - stretching vibration of the PVC vinyl group and stretching of the benzene ring in lignin (Wang et al. 2019). The peak at $1024 \mathrm{~cm}^{-1}$ was assigned to the $-\mathrm{C}-\mathrm{O}$ vibration because of the bridging crosslinking of free radicals and oxygen (Cavdar et al. 2018). The band ranging from 760 to $550 \mathrm{~cm}^{-1}$ was assigned to -C$\mathrm{Cl}$ stretching. Those bands decreased after aging, which indicated that both PVC and lignin degraded on the weathered WPC surface (Andrady et al. 2019), and the degraded composites are vulnerable to further degradation because the photo-labile carbonyl groups. The $\mathrm{C}=\mathrm{C}$ double bond groups also increased after weathered, which may be due to PVC degradation and plant fibers removal from the weathered surface. However, weathered composites did not show any significant difference $(\mathrm{p}<0.00)$, except for eucalyptus, which had the highest content of carbonyl groups.

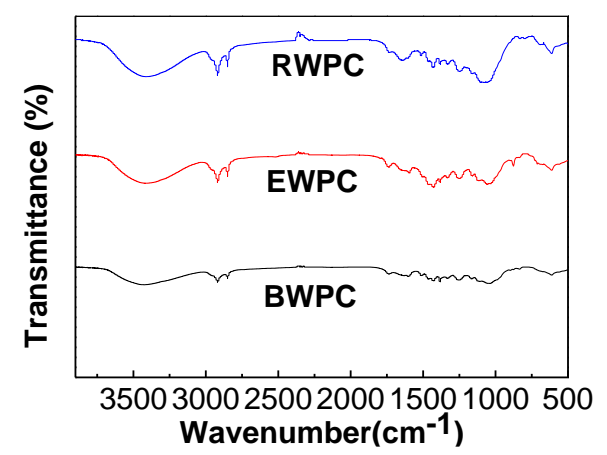

(a) Unweathered WPC

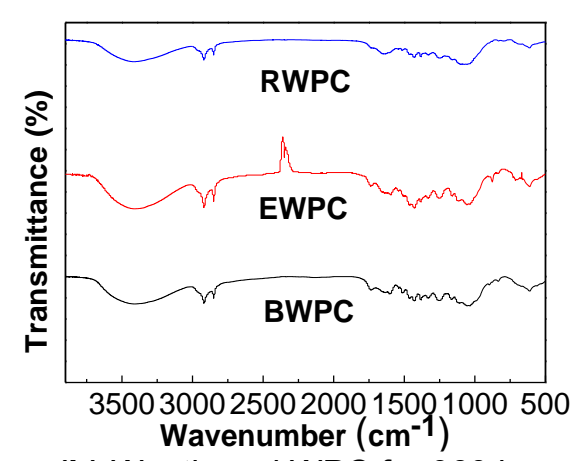

(b) Weathered WPC for $960 \mathrm{~h}$

Fig. 1. FTIR spectra of the composites Rice husk wood-plastic composites (RWPC), eucalyptus wood-plastic composites (EWPC), and bamboo wood-plastic composites (BWPC)
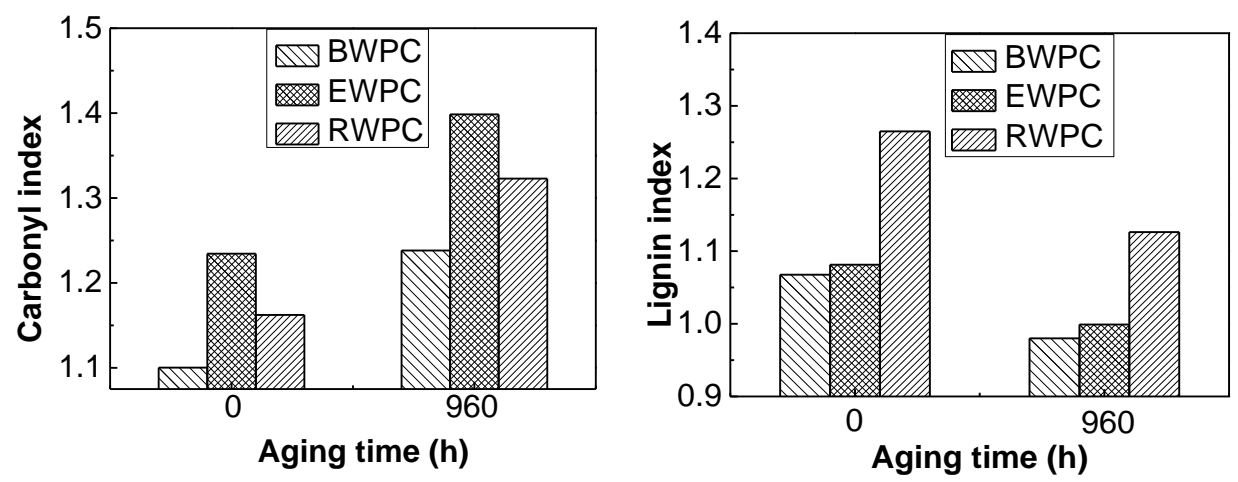

Fig. 2. Carbonyl and lignin indices of the composites

In order to further explore the degradation of the material surface, the values of carbonyl index (CID) and lignin index (LID) of all the samples were calculated. As shown in Fig. 2, the CID of the three composites increased significantly, whereas LID decreased. The CID of BWPC, EWPC, and RWPC increased 12.54\%, 13.29\%, and $13.81 \%$, respectively, and the LID decreased $8.21 \%, 7.61 \%$, and $10.96 \%$, respectively. After weathering, an increase in the content of carbonyl group reflects the degree of degradation of the WPC due to three reasons. First, the dehydrochlorination of PVC subsequently formed a carbonyl group. Second, the lignin contained an aromatic structure and chromophoric group that absorbs ultraviolet light and undergoes photodegradation to form 
a product containing a carboxyl and carbonyl groups. Finally, a cellulose macromolecule reacts with the oxygen to form cellulose peroxide and hydroperoxide, which decompose to form a ketone and thus enhances the carbonyl peak intensity (Nguyen et al. 2020). The LID was decreased because the photo-degradation reaction of lignin generated aromatic free radicals; the free radicals continue to be oxidized to form new chromophores and yields quinone type compounds (Cherian et al. 2013). Among the WPCs studied, the CID of the EWPC increased the least, whereas the LID decreased the least, indicating that EWPC was least affected by weathering.

\section{Color Change}

Table 3 lists the color change of the WPC surface. The data in Table 3 suggests that as the aging time was increased, the WPC faded significantly, and $\Delta E$ and $\Delta L$ gradually increased. It can be seen that EWPC had the least $\Delta E$ and $\Delta L$ values. Total color change of WPC was ranked as follows: EWPC $<$ BWPC $<$ RWPC. In addition, the surface lightness was ranked essentially the same as that of the total color change pattern.

Table 3. Color Change of Composites

\begin{tabular}{|c|c|c|c|c|c|}
\hline \multirow{2}{*}{ ID } & Aging Time (h) & $\Delta a^{*}$ & $\Delta b^{*}$ & $\Delta L^{*}$ & $\Delta E$ \\
\hline \multirow{3}{*}{ EWPC } & 320 & -2.82 & -4.00 & 4.81 & 6.86 \\
\cline { 2 - 6 } & 640 & -3.33 & -4.29 & 22.39 & 23.04 \\
\cline { 2 - 6 } & 960 & -5.12 & -6.51 & 25.07 & 26.40 \\
\hline \multirow{3}{*}{ BWPC } & 320 & -3.51 & -4.41 & 5.14 & 7.62 \\
\cline { 2 - 6 } & 640 & -5.14 & -6.51 & 24.34 & 25.71 \\
\cline { 2 - 6 } & 960 & -5.51 & -6.75 & 28.22 & 29.53 \\
\hline \multirow{3}{*}{ RWPC } & 320 & -3.51 & -4.58 & 5.49 & 7.96 \\
\cline { 2 - 6 } & 640 & -5.35 & -6.72 & 25.33 & 26.75 \\
\cline { 2 - 6 } & 960 & -5.85 & -7.02 & 29.20 & 30.60 \\
\hline
\end{tabular}

Previous studies (Oltean et al. 2008) have shown that the main chemical components that suffer photodegradation in plant fibers during aging are lignin and extractives, whereas the final product is quinone-type compounds, which are responsible for the color change of WPC. The degradation is accompanied by a decrease in the aromatic structural units and an increase in the conjugated carbonyl groups, which is consistent with the above FTIR analysis. The absorption of light waves by the quinone compounds and aromatic compounds in the original lignin produces discoloration of the material from a macro perspective.

During the WPC aging, $\Delta a^{*}$ and $\Delta b^{*}$ were negative values, indicating that the WPC turns blue and green. The gradual color change to blue with time might be due to the degradation of lignin into a new carbonyl type compounds, whereas the gradual color change to green was because of changes in the lignin structure (Li et al. 2009). Furthermore, during the aging process, the water present in fibers soaks and flushes the solubles (some of which affect the fiber color), causing the material to discolor. Simultaneously, the erosion of water results in the shedding of oxidized substances on its surface, internal materials continue to be affected by the radiation of light, and fading of the composite material is further aggravated. Table 3 suggests that the color of the composite surface changes rapidly during the early aging stage, which then changes slowly with the extension of aging time. This is because polyphenol extraction affects the photodegradation degree of the material, leading to a rapid decomposition of lignin on the 
surface of the exposed material at an early stage (Song et al. 2018). Various plant fibers revealed significant difference in the color change of WPC after aging $(p<0.05)$.

\section{Mechanical Properties of Composites}

The mechanical properties of the composites are shown in Fig. 3. After weathering for $960 \mathrm{~h}$, the mechanical properties of WPC were decreased. The flexural and tensile strength retention rates of EWPC, BWPC, and RWPC were $24.6 \%, 11.6 \%$, and $13.7 \%$, respectively, and $24.4 \%, 21.6 \%$, and $42.0 \%$, respectively. The mechanical properties of WPC produced significant changes upon weathering. These variations were mainly due to the chemical changes in the surface and internal oxidation, e.g., the surface oxidation process of the composite and photodegradation of plant fiber and PVC occur. These two factors would act synergistically through surface erosion and increased wettability, thus leading to the loss of mechanical properties and reduction of their service life (Chen et al. 2020).
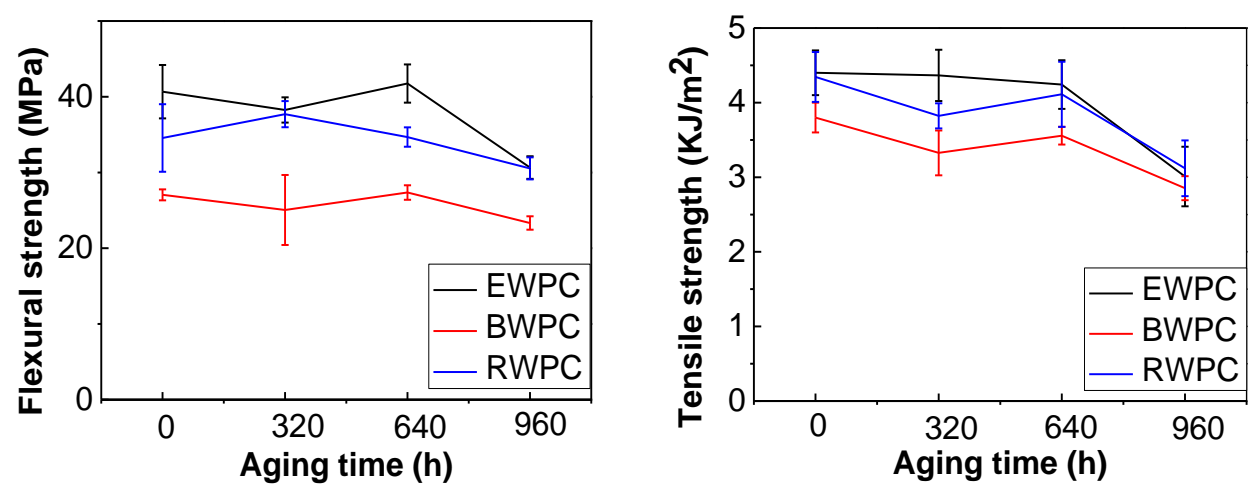

Fig. 3. Mechanical properties of composites

There were differences in the mechanical properties of the three plant species-based WPCs, of which EWPC exhibited the best comprehensive mechanical properties. This may be because of the chemical composition of plant fibers that affects the basic properties of WPC. Eucalyptus has the highest lignin content of the three plant fibers studied; therefore, EWPC exhibited the strongest interfacial adhesion (lignin hydrophilic ability is the weakest among lignin, cellulose, and hemicellulose components) and antioxidant capacity (the structure of hindered phenols in lignin can inhibit the photodegradation of plastic matrix), resulting in the best mechanical retention after aging (Chen et al. 2020)

\section{Tensile Sectional Microstructure of Composites}

Figure 4 shows the SEM images of tensile section of WPCs before and after aging for $960 \mathrm{~h}$. Figure 4 suggests that the WPC was tightly bound to the PVC matrix before aging. The PVC wraps the fiber well and makes the cross-sectional surface rough, and the PVC matrix occasionally sticks to the fiber. The mechanical properties were better, among which EWPC was the best. After aging, the combination of plant fiber and PVC was loose (Fig. 4) and the fiber was pulled out occasionally; there were little gaps and large holes between the fiber and PVC matrix. This shows that the bonding performance of fiber and PVC had deteriorated. It can be seen that the aging affected the combination of the twophase interface of WPC, which is consistent with the deterioration of the mechanical properties mentioned above. 
The reason for this phenomenon is that the thermal expansion caused by the water absorption of the composite material will further make the gap between the plant fiber and the PVC matrix become larger. Thus, the interface bonding ability decreases and the mechanical properties starts to decline. The increase in water absorption is consistent.

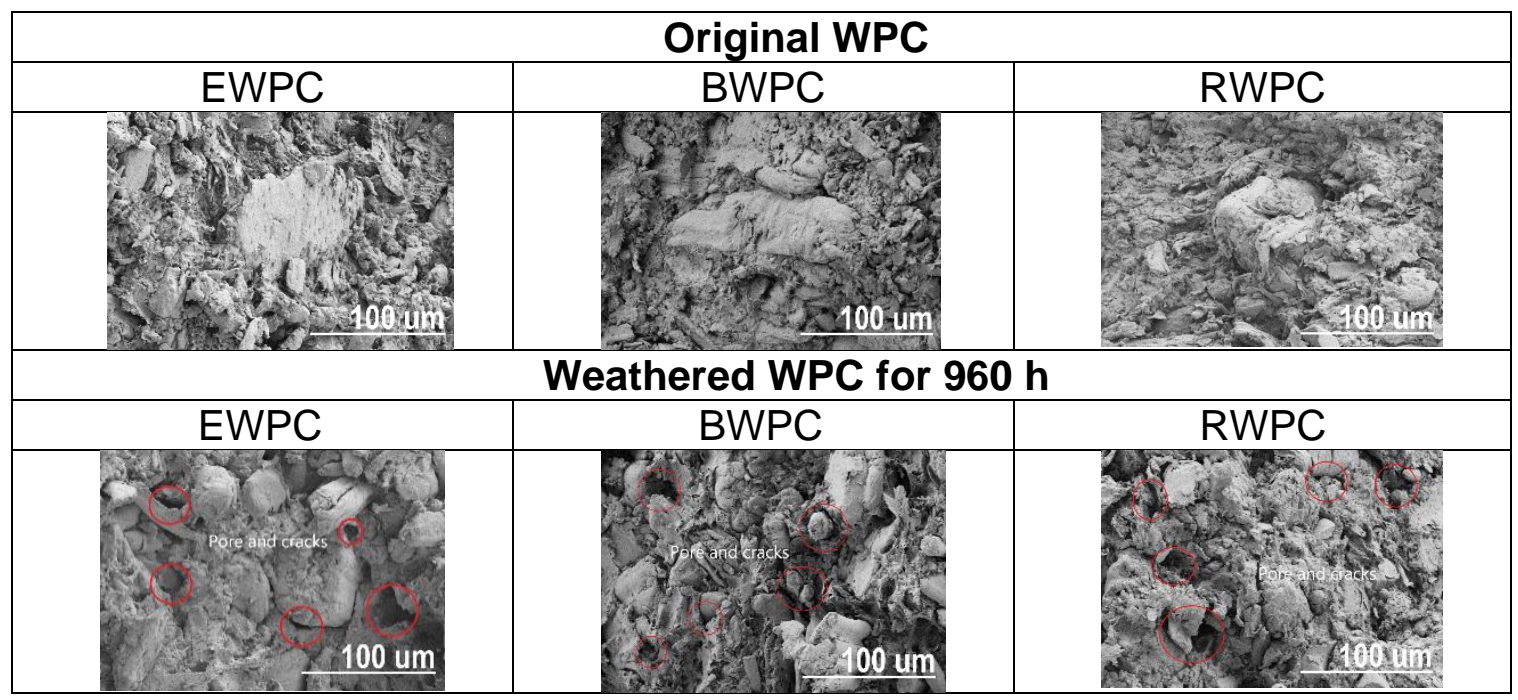

Fig. 4. SEM images of tensile section of composites (Qi et al. 2019)

\section{4-h Water Absorption of Composites}

Table 4. 24-h Water Absorption and Thickness Swelling Ratio of Composites

\begin{tabular}{|c|c|c|c|c|c|c|c|c|}
\hline \multirow{2}{*}{ ID } & \multicolumn{3}{|c|}{ Water absorption (\%), aging time (h) } & \multicolumn{4}{c|}{ Swelling ratio (\%), aging time (h) } \\
\cline { 2 - 9 } & 0 & 320 & 640 & 960 & 0 & 320 & 640 & 960 \\
\hline EWPC & 1.85 & 1.99 & 3.04 & 5.36 & 3.14 & 3.21 & 3.48 & 3.72 \\
\hline BWPC & 5.23 & 5.34 & 5.76 & 6.68 & 3.32 & 3.36 & 3.53 & 3.93 \\
\hline RWPC & 2.09 & 2.64 & 3.65 & 6.18 & 3.16 & 3.29 & 3.45 & 3.79 \\
\hline
\end{tabular}

Table 4 shows the 24-h Water absorption (WA) data and swelling radio (SR) of WPC, which increased upon weathering. Amongst the WA of the WPCs, un-weathered EWPC was minimal, whereas that of BWPC was the largest. Water absorption depends on the $-\mathrm{OH}$ of the fiber, amorphous areas, micro vides in the material and the state of adhesion at the fiber-matrix interface (Law and Mohd 2011). And precisely for BWPC, bamboo contains more hemicelluloses and therefore more amorphous areas. Compared with other composite materials, BWPC exhibited the highest water absorption and lowest interfacial adhesion, which can be clearly seen from the mechanical test results. After weathering, the WA and SR were increased, but those of EWPC showed the smallest changes. Combining tensile fracture surface analysis and mechanical properties, it can be seen that EWPC had better interface bonding. Attributed to the presence of more lignin in eucalyptus fiber, it can be used as an antioxidant to slow down the aging of the composites (Tamrakar et al. 2011). Another effect of the lignin was to reduce the cracks on the surface and the irreversible damage caused by repeated water absorption of the weathered composites, thereby reducing the water absorption and swelling radio of them (Erdogan and Huner 2018) 


\section{CONCLUSIONS}

In this study, the influence of plant species on the chemical modification and physical properties (water absorption, color change, mechanical properties, and tensile sectional microstructure) of wood-polymer composite (WPC) were examined for a polyvinyl chloride (PVC) matrix filled with the fibers of either eucalyptus wood, rice husk, or bamboo.

1. The water absorption, swelling ratio, color change, and surface chemical oxidation of all the composites increased upon longer exposure to weathering agents. There were significant differences in the carbonyl index, lignin index, color change, and water absorption values of weathered composites among various plant species. Meanwhile, the bonding performance of plant fiber and PVC deteriorated. The prominent changes in chemistry, with respect to carbonyl groups hydroxyl groups, were observed in all the three plant species-based WPCs.

2. Eucalyptus can be preferred for applications in which color stability and mechanical properties are important. After weathering for $960 \mathrm{~h}$, compared with the original WPC, the tensile strength and flexural strength of EWPC decreased $24.4 \%$ and $24.6 \%$, respectively, lignin index decreased $8.2 \%$, carbonyl index increased $12.5 \%$, and the interface between plant fiber and PVC deteriorated. Generally, plant species play a major role in the composite's performance of the respective WPC, suggesting that careful selection of plants can improve the anti-aging properties.

\section{ACKNOWLEDGEMENTS}

This work was supported by the Regional Cooperative Innovation in Autonomous Region (2019E0241), and the Natural Science Foundation of the Jiangsu Higher Education Institutions of China (Grant No. 18KJD430002).

\section{REFERENCES CITED}

Andrady, A. L., Pandeyb, K. K., and Heikkiläc, A. M. (2019). "Interactive effects of solar UV radiation and climate change on material damage," Photochemical \& Photobiological Science 18, 804-825. DOI: 10.1039/c8pp90065e

Cavdar, A. D., Tomak, E. D., and Mengelolu, F. (2018). "Long-term leaching effect on decay resistance of wood-plastic composites treated with boron compounds," Journal of Polymer Environment 26, 756-764. DOI: 10.1007/s10924-017-0992-7

Chan, C. M., Vandi, L., Pratt, S., Halley, P., Richardson, D., Werker, A., and Laycock, B. (2020). "Mechanical stability of polyhydroxyalkanoate (PHA)-based wood plastic composites (WPCs)," Journal of Polymers and the Environment 28(5) 1571-1577. DOI: 10.1007/s 10924-020-01697-9

Chen, B., Luo, Z., Chen, H., Chen, C., Cai, D., Qin, P., Cao, H., and Tan T. (2020). "Wood Plastic composites from the waste lignocellulosic biomass fibers of bio-fuels processes: A comparative study on mechanical properties and weathering effects," Waste and Biomass Valorization 11, 1701-1710. DOI: 10.1007/s12649-018-0413-8

Cherian, B., Leo, A., Chaves, M., and Narine, S. (2013). "Environmental ageing studies 
of chemically modified micro and nano-fibril phenol formaldehyde composites," Industrial Crops and Products 49, 471-483. DOI: 10.1016/j.indcrop.2013.04.033

Dou, L. Y., Wang, M., and Chen, C. (2015). "The research advancement and application status of wood-plastics composites in Mainland," Science and Technology Vi-Sion 14, 103-104. DOI: 10.19694/j.cnki.issn2095-2457.2015.14.075

Erdogan, S., and Huner, U. (2018). "Physical and mechanical properties of PP composites based on different types of lignocellulosic fillers," Journal of Wuhan University of Technology-Materials Science Edition 33, 1298-1307. DOI: 10.1007/s11595-0181967-9

Fabiyi, J., and McDonald, A. (2010). "Effect of wood species on property and weathering performance of wood plastic composites," Composites Part A: Applied Science and Manufacturing 41(10), 1434-1440. DOI: 10.1016/j.compositesa.2010.06.004

Gao, K., Shi, H., Sun, B., Wang, Z., Yang, Z., Xing, Y., and Yang, Y. (2016). "Effects of hydro-thermal aging on properties of glass fiber / epoxy composites," Acta Materiae Compositae Sinica 33(6), 1147-1152. DOI: 10.13801/j.cnki.fhclxb.20160108.001

GB/T 9431 (2008). "Plastics," Standardization Administration of China, Beijing, China.

GB/T 1040.1 (2006). "Plastics: Determination of tensile properties Part1: General principles," Standardization Administration of China, Beijing, China.

GB/T 16422.3 (2014). "Plastics- Methods of exposure to laboratory light sources- Part3: Fluorescent UV lamps," Standardlization Administration of China, Beijing, China.

GB/T 17657 (2013). "Test methods of evaluating the properties of wood-based panels and surface decorated wood-based panels," Standardization Administration of China, Beijing, China.

Guan, Q. Y., and Li, W. P. (2018). "Effect of hydro-thermal condition on typical mechanical property of 7781/CYCOM 7701 fiberglass/epoxy composite," Acta Materiae Compositae Sinica 35(12), 3288-3297. DOI: 10.13801/j.cnki.fhclxb.20180319.001

Krzysztof, M., Magdalena, S., Rafal, M., Tomasz, K., Bartlomiej, J., and Piotr, R. (2019). "The effect of accelerated aging on polylactide containing plant extracts," Polymers 11(4), Article Number 575. DOI: 10.3390/polym11040575.

Law, T. T., and Mohd Z., A. (2011). "Water absorption and dimensional stability of short kenaf fiber-filled polypropylene composites treated with maleated polypropylene," Journal of Applied Polymer Science 120, 563-572 DOI: 10.1002/app.33184

Li, Y. F., Liu, Y. X., Yu, H. P., and Sun, Q. (2009). "Property improvement of wood polymer composites with glycidyl methacrylate," Acta Materiae Compositae Sinica 26(5), 1-7. DOI: 10.13801/j.cnki.fhclxb.2009.05.028

Nguyen, V., Nguyen, T., Zhang, A., Hao, J., and Wang, W. (2020). "Effect of three tree species on UV weathering of wood flour-HDPE composites," Journal of Forestry Research 31(3) 1071-1079. DOI: 10.1007/s11676-019-00890-4

Oltean, L., Teischinger, A., and Hansmann, C. (2008). "Wood surface discoloration due to simulated indoor sunlight exposure," Holz als roh-und Werkstoff 66(1), 51-56. DOI: 10.1007/s00107-007-0201-9

Paul, F., Benjamin, E., and Dawson, A. (2009). "Colorimetric and vibrational spectroscopic characterization of weathered surfaces of wood and rigid polyvinyl chloride-wood flour composite lumber," Wood Science and Technology 43(7-8), 669678. DOI: $10.1007 / \mathrm{s} 00226-009-0254-5$

Peng, Y., Liu, R., Cao, J., and Chen, Y. (2014). "Effects of UV weathering on surface properties of polypropylene composites reinforced with wood flour, lignin, and 
cellulose," Applied Surface Science 317, 385-392. DOI:

10.1016/j.apsusc.2014.08.140

Petchwattana, N., Sanetuntikul, J., Sriromreun, P., and Narupai, B. (2017). "Wood plastic composites prepared from biodegradable poly (butylene succinate) and Burma padauk sawdust (Pterocarpus macrocarpus): Water absorption kinetics and sunlight exposure investigations," Journal of Bionic Engineering 14, 781-790. DOI: 10.1016/S1672-6529(16)60443-2

Qi, R., He, C., and Jin, Q. (2019). "Effect of acrylate-styrene-acrylonitrile on the aging properties of eucalyptus/PVC wood-plastic composites," BioResources 14(4), 91599168. DOI: 10.15376/biores.14.4.9159-9168

Song, H. S., Wang, M., Lu, X. F., Wei, T., and Guo, J. (2018). "Effect of thermaloxidative aging on static and dynamic mechanical properties of long glass fiber reinforced nylon 10T composites," Acta Materiae Compositae Sinica 33(10), 21582165. DOI: 10.13801/j.cnki.fhclxb.20160127.002

Tamrakar, S., Roberto A., and Lopez A. (2011). "Water absorption of wood polypropylene composite sheet piles and its influence on mechanical properties," Construction Building Mater 25(10), 3977-3988 DOI: 10.1016/j.conbuildmat.2011.04.031

Temiz, A., Terziev, N., Eikenes, M., and Hafren, J. (2007). "Effect of accelerated weathering on surface chemistry of modified wood," Applied Surface Science 253(12), 5355-5362. DOI: 10.1016/j.apsusc.2006.12.005

Wang, H., Zhang, J., Fu, H., and Wang Q. (2019). "Effect of an antioxidant on the life cycle of wood flour/polypropylene composites," Journal of Forestry Research 11 1435-1443. DOI: 10.1007/s11676-019-00898-w

Article submitted: March 7, 2021; Peer-review completed: May 15, 2021; Revised version received and accepted: May 25, 2021; Published: June 4, 2021.

DOI: 10.15376/biores.16.3.5261-5271 